\title{
Transcriptomic Analysis of Vibrio parahaemolyticus Reveals Different Virulence Gene Expression in Response to Benzyl Isothiocyanate
}

\author{
Jie Song ${ }^{1}$, Hong-Man Hou ${ }^{1}$, Hong-Yan Wu ${ }^{2}$, Ke-Xin Li ${ }^{1}$, Yan Wang ${ }^{1}$, Qian-Qian Zhou ${ }^{1}$ \\ and Gong-Liang Zhang ${ }^{1, * \mathbb{D}}$ \\ 1 School of Food Science and Technology, Dalian Polytechnic University, Dalian 116034, China; \\ sj101521@163.com (J.S.); houhongman2011@hotmail.com (H.-M.H.); LiKexin0903@hotmail.com (K.-X.L.); \\ 18804208629@163.com (Y.W.); 15940283178@163.com (Q.-Q.Z.) \\ 2 Graduate School of Environmental and Life Science, Okayama University, Okayama 700-8530, Japan; \\ wuhongyan1908@hotmail.com \\ * Correspondence: zgl_mp@163.com; Tel.: +86-411-86322020
}

Received: 1 February 2019; Accepted: 19 February 2019; Published: 20 February 2019

\begin{abstract}
Vibrio parahaemolyticus isolated from seafood is a pathogenic microorganism that leads to several acute diseases that are harmful to our health and is frequently transmitted by food. Therefore, there is an urgent need for the control and suppression of this pathogen. In this paper, transcriptional analysis was used to determine the effect of treatment with benzyl isothiocyanate (BITC) extracted from cruciferous vegetables on $V$. parahaemolyticus and to elucidate the molecular mechanisms underlying the response to BITC. Treatment with BITC resulted in 332 differentially expressed genes, among which 137 genes were downregulated, while 195 genes were upregulated. Moreover, six differentially expressed genes (DEGs) in RNA sequencing studies were further verified by quantitative real-time polymerase chain reaction (qRT-PCR). Genes found to regulate virulence encoded an L-threonine 3-dehydrogenase, a GGDEF family protein, the outer membrane protein $\mathrm{OmpV}$, a flagellum-specific adenosine triphosphate synthase, TolQ protein and VirK protein. Hence, the results allow us to speculate that BITC may be an effective control strategy for inhibiting microorganisms growing in foods.
\end{abstract}

Keywords: Vibrio parahaemolyticus; benzyl isothiocyanate; virulence; RNA sequencing; qRT-PCR

\section{Introduction}

V. parahaemolyticus is a Gram-negative, highly motile, halophilic bacterium that is always naturally found in marine environments and commonly isolated from seafood, including fish, crop, shrimp, scallops and oysters [1] due to water-feeding activity. Among the most important food-borne pathogens, $V$. parahaemolyticus can lead to a variety of symptoms, such as headache, fever, nausea, vomiting and watery diarrhea, within $24 \mathrm{~h}$ of infection [2]. In a report, on the eastern coast of China $V$. parahaemolyticus has caused $41 \%$ of food-borne disease outbreaks [3]. Moreover, V. parahaemolyticus is one of most severe food-borne pathogens that can spectacularly increase the mortality rate of aquatic life, and seriously infect humans [4]. Therefore, it is necessary to develop a wide variety of control measures to suppress $V$. parahaemolyticus.

Antibiotics have been used in aquaculture for a long time, however, after prolonged use the antibiotics accumulate in the seafood and result in bacteria gaining antibiotic resistance, and a large amount of antibiotics can even induce aplastic anemia in humans [5]. To help solve these situations, other kinds of food additives are commonly used in food processing, production and packaging to maintain the quality of food and limit the growth of pathogens. Many studies have 
concentrated on seeking new alternatives to prevent and treat food-borne pathogens. A previous report demonstrated that essential oils (EOs) from ginger and mustard could inhibit the growth of $V$. parahaemolyticus at $5{ }^{\circ} \mathrm{C}$ storage [6]. Feng et al. used RNA sequencing to prove that aqueous ozone could inhibit almost all genes in $V$. parahaemolyticus to kill the bacteria [7]. Formed from the digestion of glucotropaeolin in cruciferous vegetables, benzyl isothiocyanate (BITC) displays inhibitory effects against cancer due to glucosinolates. BITC can release biologically active isothiocyanates (ITCs) that play a main role in reducing carcinogens [8]. Previous studies mainly focused on the antibacterial and antifungal effects of BITC $[9,10]$. Dufour et al. demonstrated that BITC had the antimicrobial activities against $V$. parahaemolyticus [10]. However, no research has been conducted on the effect of BITC on $V$. parahaemolyticus at the transcriptomic level.

Thermostable direct hemolysin (TDH) is recognized as a major virulence factor of $V$. parahaemolyticus in causing human gastrointestinal disorders [11]. Previous reports have shown that the tdh gene is strongly related to clinical strains [12]. Banu et al. found that essential oils from Cinnamomum tamala could mediate the virulence factors of $V$. parahaemolyticus by influencing its biofilm, polysaccharides, lipopolysaccharides, flagellum and cytotoxins [13]. Therefore, the genes encoded by these proteins mentioned above are important for studying virulence factors. An effective tool for investigating pathogenesis is transcriptome analysis, which has widely been used to investigate gene expression changes, and to identify pathways that may be affected. Natural compounds that are potentially antimicrobial can provide novel and meaningful methods for inhibiting the growth of pathogens.

Hence, the purpose of this paper was to determine the differential transcriptional expression of the virulence genes of $V$. parahaemolyticus treated with BITC by means of RNA sequencing and to verify the expressions of significantly regulated genes (Table 1) by quantitative real-time polymerase chain reaction (qRT-PCR).

Table 1. Primers used to verify gene expression level by quantitative real-time polymerase chain reaction (qRT-PCR).

\begin{tabular}{ccc}
\hline Gene & Primer & Sequence $\left(5^{\prime} \rightarrow \mathbf{3}^{\prime}\right)$ \\
\hline \multirow{2}{*}{$16 S$ r $R N A$} & $16 \mathrm{~S}$ rRNA-F & TATCCTTGTTTGCCAGCGAG \\
& $16 \mathrm{~S}$ rRNA-R & CTACGACGCACTTTTGGGA \\
$t d h$ & tdh-F & GGCATTTGGATGACCGAAGTA \\
& tdh-R & CTGACCAATCGCAACCACTTC \\
$V P A 0318$ & VPA0318-F & AGGTTACTTAGCGGGTGCG \\
& VPA0318-R & TTCACGGTCTTTGATGCC \\
$f l i I$ & fliI-F & TGCGGAACCCATCAACCC \\
& fliI-R & CGTCCGTCTTCGCCCAAA \\
$V P 1057$ & VP1057-F & CGGTTCAATCAGCCCATAC \\
& VP1057-R & AACGCTTCTGCGATACCTG \\
$V P A 0243$ & VPA0243-F & AACGCTTCTGCGATACCTG \\
& VPA0243-R & TTGCCATAGTGCGTCGTAGTCG \\
$V P A 0202$ & VPA0202-F & CGAAGAAGTGATGGTGGTG \\
& VPA0202-R & CTCGCATTGGTGAGTTGACG \\
\hline
\end{tabular}

\section{Results}

\subsection{Antibacterial Tests}

By measuring the diameter of the inhibition zone ( $\mathrm{mm}$ ) of different sulfur compounds, we selected BITC as the inhibitor because it had the strongest antibacterial effect, with a minimum inhibitory concentration (MIC) of $9.54 \mu \mathrm{mol} / \mathrm{L}$.

\subsection{Global Changes at Transcriptome Level}

Transcriptome analysis is a powerful tool for revealing the molecular mechanisms underlying the response of pathogens to food additives. Illumina RNA deep sequencing (RNA sequencing) technology 
generated two transcriptomic databases to further understand the pathogenicity of $V$. parahaemolyticus. We obtained a total $9.81 \mathrm{~Gb}$ of sequencing data, including 70,210,670 raw reads and 65,318,934 clean reads, with all the data having an average error rate of less than $0.02 \%$. After removing low-quality and adapter sequences, 11,409,316, 12,152,830, 11,803,196 and 12,650,370, 9,990,268, 12,204,690 clean reads were obtained for C_BITC and Q_BITC samples, respectively. The Q20 and Q30 percentages were higher than $96 \%$ and $92 \%$, respectively. In the C_BITC and Q_BITC samples, the average GC percentages were $48.29 \%, 48.39 \%, 48.35 \%$ and $48.20 \%, 48.01 \%, 48.08 \%$, respectively. An overview of the transcriptome assembly statistics is shown in Table 2.

Table 2. Summary of the RNA sequencing data.

\begin{tabular}{|c|c|c|c|c|c|c|c|}
\hline Sample Name & Raw Reads & Clean Reads & Clean Bases (Gb) & Error $(\%)$ & Q20 (\%) & Q30 (\%) & GC (\%) \\
\hline C_BITC2 & $12,152,830$ & $11,176,478$ & 1.68 & 0.02 & 96.93 & 92.11 & 48.39 \\
\hline Q_BITC1 & $12,650,370$ & $11,915,336$ & 1.79 & 0.02 & 97.06 & 92.39 & 48.20 \\
\hline Q_BITC2 & $9,990,268$ & $9,170,324$ & 1.38 & 0.02 & 97.05 & 92.36 & 48.01 \\
\hline Q_BITC3 & $12,204,690$ & $11,470,672$ & 1.72 & 0.02 & 97.14 & 92.53 & 48.08 \\
\hline
\end{tabular}

Q20: percentage of bases with a Phred value $>20$; Q30: percentage of bases with a Phred value $>30$.

During V. parahaemolyticus growth in 1/4 MIC BITC in comparison to the sample without BITC treatment, gene expression levels were assessed by RNA sequencing technology to clarify molecular mechanisms of BITC on V. parahaemolyticus. To analyze and characterize the differentially expressed genes (DEGs) following BITC treatment, a $p$-value less than 0.05 was selected as the limitation for screening DEGs. A number of genes were found to be regulated under 1/4 MIC BITC treatment. In total, 332 genes presented a FPKM > 1 compared with the Q_BITC sample, implying that these genes were differentially expressed, of which 195 were upregulated and 137 were downregulated (Figure 1).

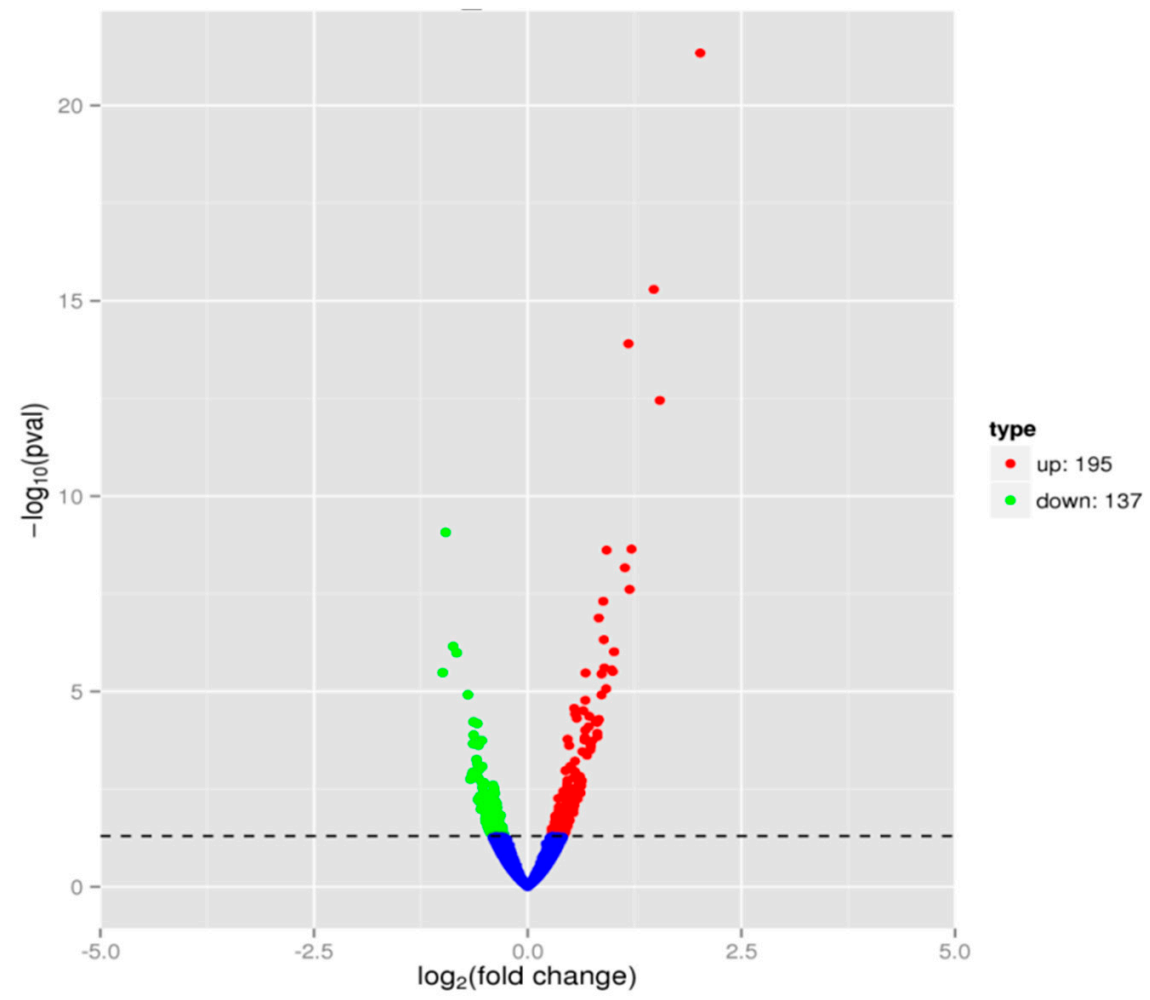

Figure 1. Volcano plot of differentially expressed genes (DEGs). In total, 332 genes present different expression levels. Red indicates upregulated expression, green indicates downregulated expression and blue indicates no significant differential expression when comparing Q_BITC with the C_BITC sample. 
From the transcriptome data, gene ontology (GO) terms consisted of 332 unigenes. GO assignments are widely used to categorize gene functions, and the results regarding the effect of BITC on the expression of different genes in V. parahaemolyticus are displayed (Figure 2). Three domains, including biological process (10 subcategories), cellular component (8 subcategories) and molecular function (12 subcategories), comprised 30 major GO terms.

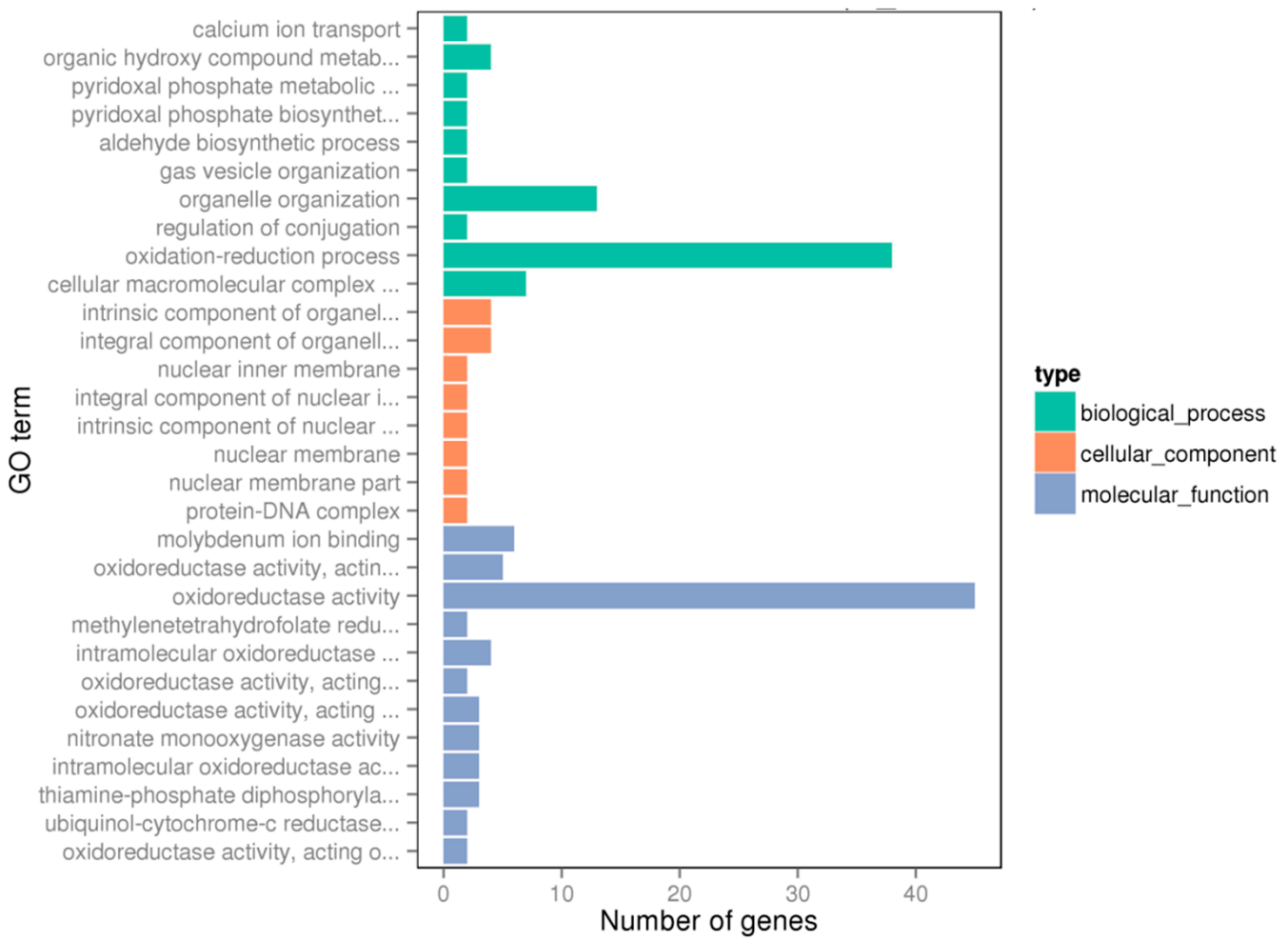

Figure 2. Functional enrichment of differentially expressed genes on gene ontology (GO) categorization.

A total of 332 genes are assigned to 30 terms.

Significant changes in the biological process included organic hydroxy compound metabolic process, oxidation-reduction process, organelle organization, and cellular macromolecular complex assembly. The major changes in cellular component included an integral component of organelle membrane, intrinsic component of organelle membrane, nuclear inner membrane, and integral component of nuclear inner membrane. Molecular function mainly comprised molybdenum ion binding, acting on single donors with oxidoreductase activity, incorporation of molecular oxygen, oxidoreductase activity, and intramolecular oxidoreductase activity (Figure 3).

\subsection{KEGG Pathway Analysis}

To identify the annotated unigenes-involved biological processes, Kyoto Encyclopedia of Genes and Genomes (KEGG) pathway analysis was carried out. In the KEGG analysis, DEGs were mapped to 65 reference pathways in KEGG database to analyze the functions of DEGs in the presence of BITC. DEGs with significant enrichment were mainly referred to 20 pathways (Figure 4), such as microbial metabolism in diverse environments, metabolic pathways, two-component systems, carbon metabolism, and biosynthesis of secondary metabolites. The results of KEGG pathway analysis revealed that bacteria treated with BITC displayed significantly enriched pathways. 


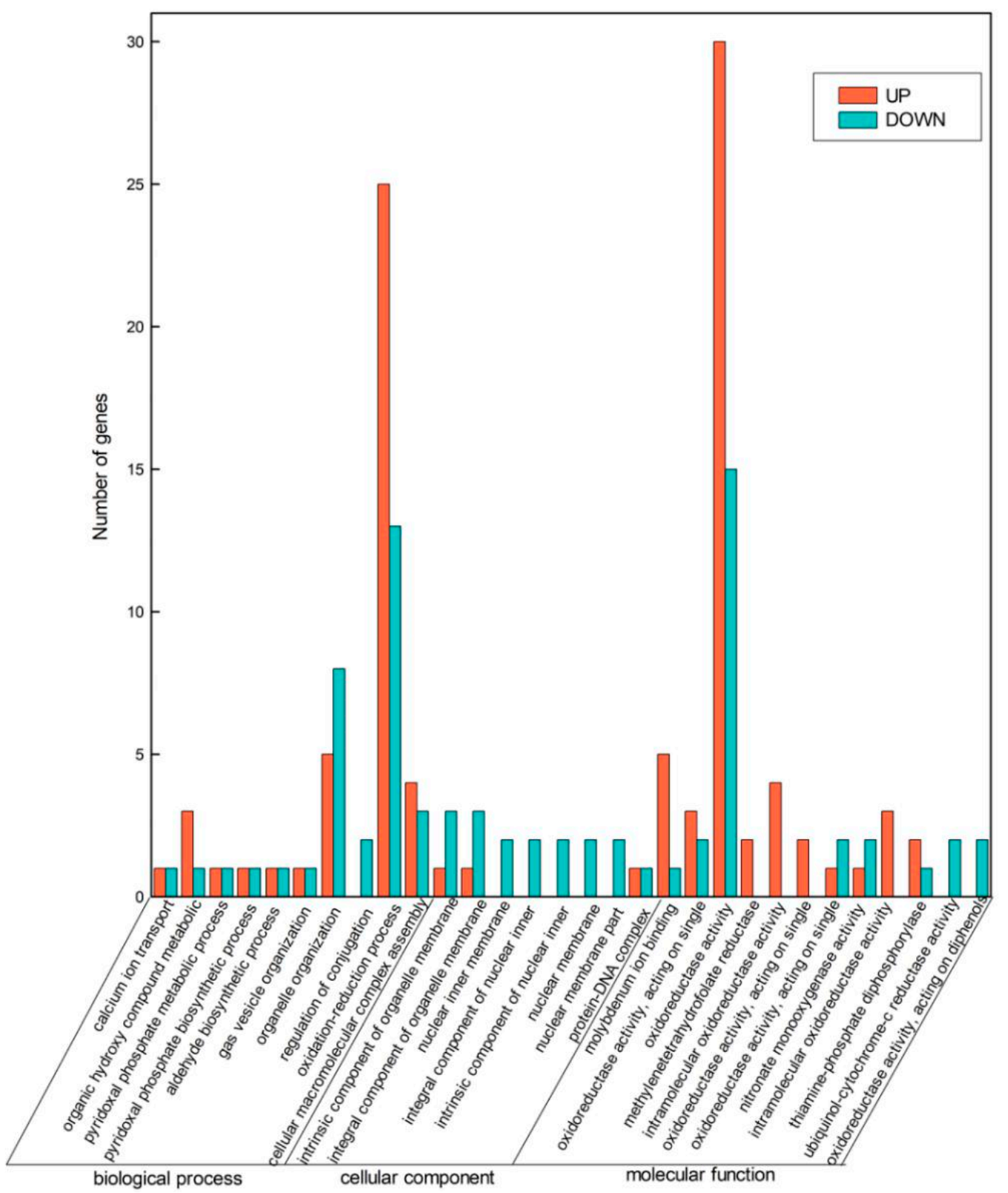

Figure 3. Transcriptomic analysis results. Differential expression of genes related to the functional categories biological process, cellular component, and molecular function of $V$. parahaemolyticus grown in the presence of benzyl isothiocyanate (BITC).

\subsection{Genes Related to Virulence and Validation the RNA Sequencing Data by qRT-PCR}

First, qRT-PCR is a powerful tool to substantiate transcriptome data and analyze the gene expression level in V. parahaemolyticus to understand its virulence and the effect of BITC on its pathogenicity. Previous studies have already demonstrated the antimicrobial potential of BITC against various pathogens, such as Escherichia coli and Campylobacter jejuni. As mentioned above, it is similar to our findings in this study that BITC has antimicrobial potential against $V$. parahaemolyticus. Genes related to virulence, including tdh, fliI, VPA0318, VP1057, VPA0243 and VPA0202 were downregulated under BITC treatment in the RNA sequencing data as shown in Table 3. Therefore, the six genes mentioned above were selected for validation using $\mathrm{PPCR}$. 


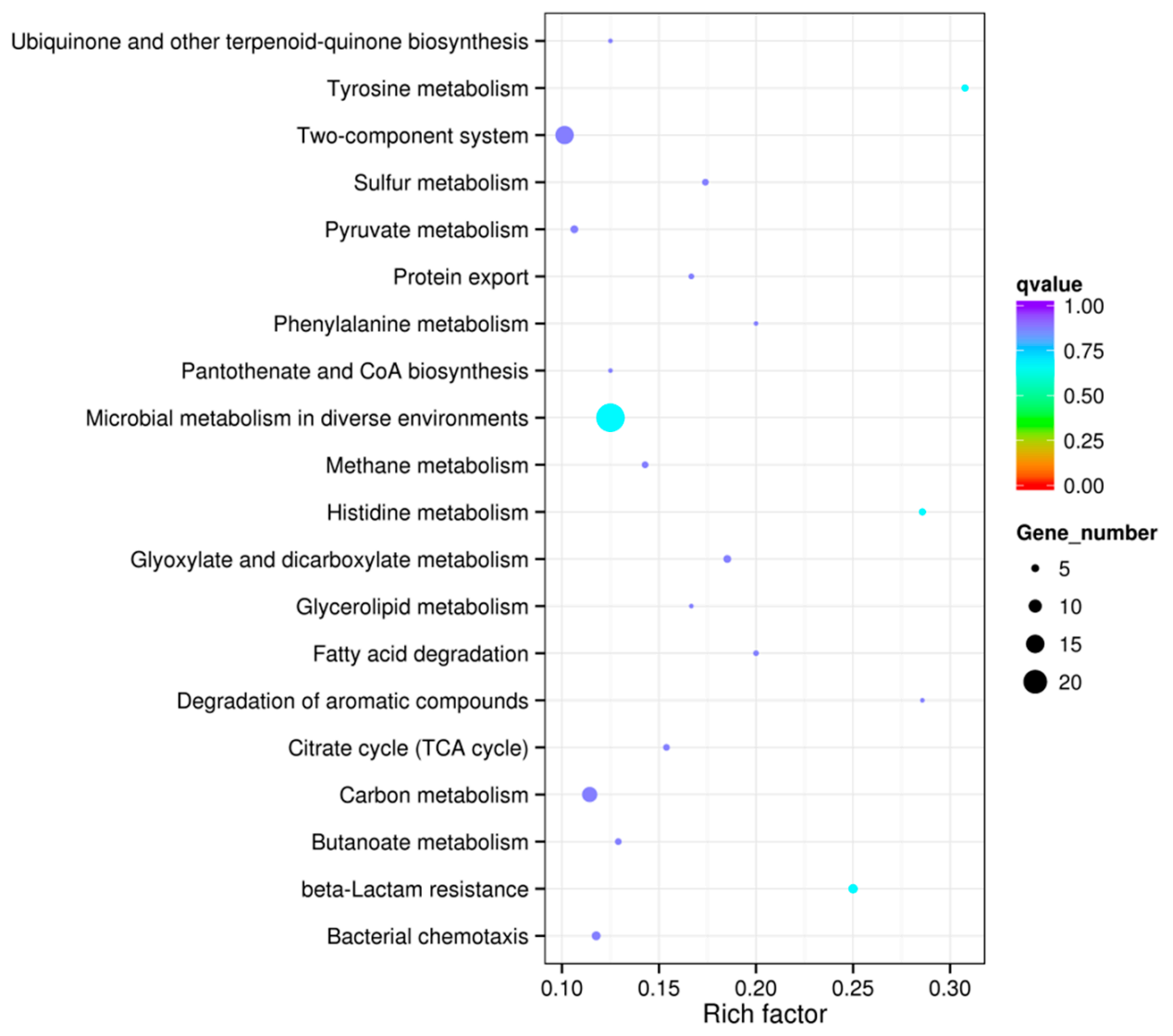

Figure 4. Kyoto Encyclopedia of Genes and Genomes (KEGG) classification of the differentially expressed genes. In total, 332 unigenes are assigned to 20 special KEGG pathways. Q value is an index used to determine the enrichment of the KEGG pathways. The closer the q value is to zero, the more significant the enrichment.

Table 3. Data of the differentially expressed genes.

\begin{tabular}{cccccc}
\hline \multirow{2}{*}{ Gene_ID } & Gene Name & $\begin{array}{c}\text { log2 Fold Change } \\
\text { (Q_BITC vs. C_BITC) }\end{array}$ & $\begin{array}{c}\text { Pval } \\
\text { (Q_BITC vs. C_BITC) }\end{array}$ & $\begin{array}{c}\text { Padj } \\
\text { (Q_BITC vs. C_BITC) }\end{array}$ & $\begin{array}{c}\text { Significant } \\
\text { (Q_BITC vs. C_BITC) }\end{array}$ \\
\hline VPA1509 & $t d h$ & -0.32858 & 0.04469 & 0.62131 & DOWN \\
VPA0318 & - & -0.95983 & $8.44 \mathrm{E}-10$ & $7.18 \mathrm{E}-07$ & DOWN \\
VP2246 & $f l i$ & -0.3946 & 0.029624 & 0.57503 & DOWN \\
VP1057 & - & -0.38624 & 0.049817 & 0.63785 & DOWN \\
VPA0243 & - & -0.49488 & 0.0045398 & 0.19316 & DOWN \\
VPA0202 & - & -0.4573 & 0.030909 & 0.58184 & DOWN \\
\hline
\end{tabular}

The results of relative gene expression for V. parahaemolyticus treated with BITC are shown in Figure 5. From the histogram of these genes expression levels, it is clear that $t d h$, the most common virulence gene, was inhibited to the maximum extent as it dropped to 0.08 with BITC treatment. Additionally, the expression levels of VPA0202, VPA0243, VP1057, fliI and VPA0318 were decreased compared with sample C_BITC. Significant downregulation in the expression of sample Q_BITC relative to sample C_BITC was exhibited in the qPCR results. Thus, the qPCR results were in accordance with our RNA sequencing data, thereby confirming those results. 


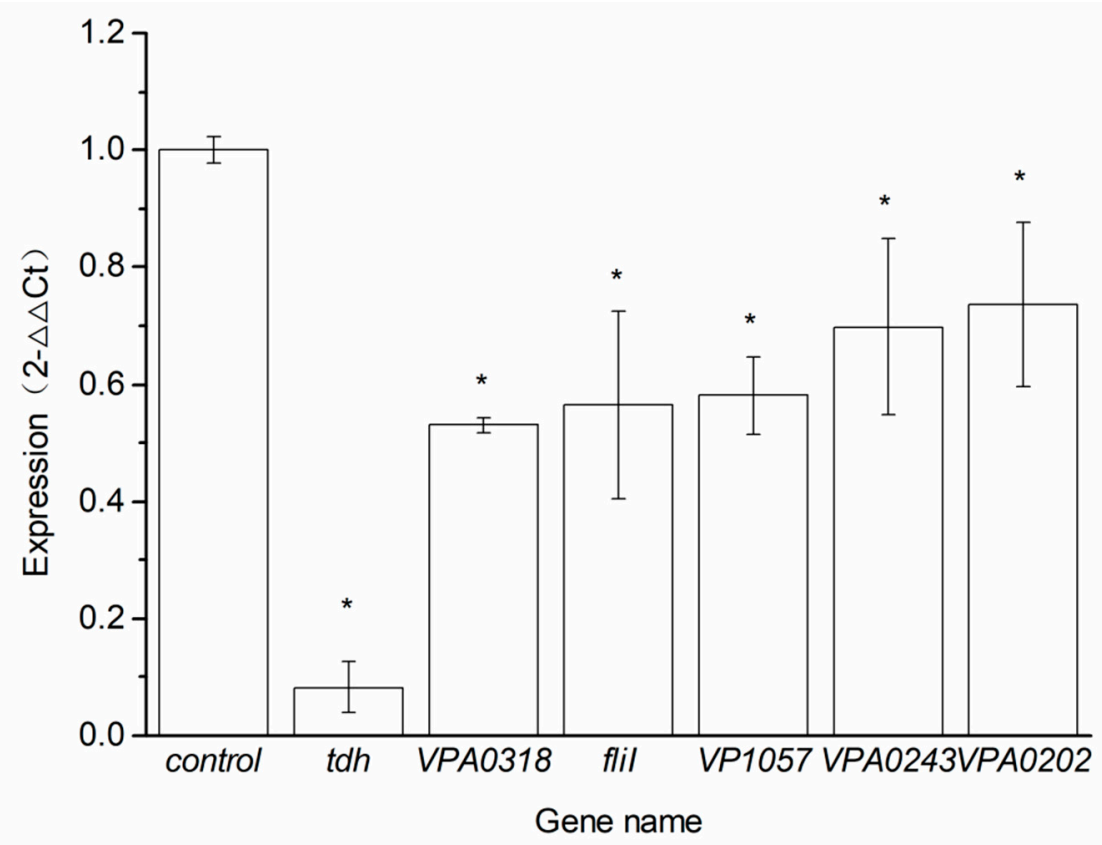

Figure 5. Validation of differentially expressed genes (DEGs) by qRT-PCR. Relative expression of $t d h$, VPA0318, fliI, VP1057, VPA0243 and VPA0202 compared with 16S rRNA of the control normalized to one. The bar results are expressed as means \pm SD from three independent replicates. * shows significant differences $(p<0.05)$.

\section{Discussion}

In regard to the antibacterial mechanism of natural products, the approach of transcriptomic analysis is useful to screen the differentially expressed genes in various pathogenic bacteria. Moreover, qRT-PCR is the direct method to validate the results of transcriptomic analysis. Therefore, several studies have combined transcriptomic analysis and qRT-PCR to explore the antibacterial mechanism in different pathogenic bacteria. Tan et al. have used RNA sequencing to screen six differentially expressed genes involved in biofilm formation of Staphylococcus aureus treated with ursolic acid, and further validated the gene expression level by qRT-PCR [14]. To explore the antibacterial mechanism of essential oil extracted from Baccharis psiadioides against Listeria monocytogenes, five differentially expressed genes associated with virulence were screened by RNA sequencing and confirmed by qRT-PCR [15]. Therefore, we investigated the antibacterial effect of BITC against $V$. parahaemolyticus at a transcriptomic level, and further studied the virulence gene expression in response to BITC treatment.

Seafood-borne illnesses and infections because of eating raw or undercooked oysters are caused by V. parahaemolyticus which is a human pathogen [16]. V. parahaemolyticus has led to severe losses in aquaculture in past years. Some available physical and chemical methods have been used to prevent and control $V$. parahaemolyticus infections in seafood [4,17]. Many kinds of food additives are widely used in the food industry to eliminate food-borne pathogens. For instance, adding grape seed extract to seawater can reduce the population of food-borne pathogens. Previous research has indicated the rapid and strong bactericidal effect of BITC on Gram-negative bacteria, while BITC can inhibit or may not affect the growth of Gram-positive bacteria [18]. Meanwhile, during the decontamination process, adding diverse amounts or concentrations will make a difference. With increasing global occurrences of $V$. parahaemolyticus, it is increasingly important to understand the virulence factors as well as the impacts on humans [17]. In this paper, we used BITC as a food additive to investigate the effect of BITC treatment on the gene expression response of $V$. parahaemolyticus. Virulence genes such as $t d h$, VPA0318, fliI, VP1057, VPA0243 and VPA0202 were downregulated $(p<0.05)$ in the presence of BITC. The major virulence factor of V. parahaemolyticus-TDH is coded by $t d h$ [14], which is closely related to 
pathogenicity. Reported by an epidemiological study, TDH occupies an important position in leading severe pathogenic ability in V. parahaemolyticus, and it appears in almost all (95\%) clinical isolates. TDH has the ability to lyse red blood cells and produce a special hemolysis ring on Wagatsuma blood agar plates [19]. 'Kanagawa phenomenon' is the phenomenon in which TDH is secreted and lyses red blood cells while simultaneously generating a hemolysis ring on Wagatsuma blood agar plates and is commonly related to gastroenteritis. Previous reports have also revealed that hemolytic activity and cytotoxicity of TDH are related to bacterial virulence.

Type III secretion systems (T3SSs) regulate the virulence of many animal and plant bacterial pathogens [20]. The peripheral membrane ATPase-FliI plays an important role in the type III protein export mechanism that depends on its bacterial flagellum [21]. Highly homologous to pathogenic bacteria type III secretion systems, FliI injects virulence effector proteins into the eukaryotic host cells for invasion [22]. The latest findings examined the flagellar adhesive and invasive properties, especially related to the flagellum are potential virulence factors [23].

From the transcriptome data, we found that the gene VPA0243 encodes VirK protein, which is reported to be related to virulence in Salmonella. Typhimurium, and this study are the first to find VirK in V. parahaemolyticus. A previous report concluded that VirK was important in late stages of Salmonella enteric fever, and for the host environment, it most likely causes the remodelling of the bacterial outer membrane. The $\triangle V P A 0243$ mutant of $S$. Typhimurium lessens virulence in a mouse infection model and decreases survival in macrophages [24]. In agreement with this, Spencer et al. demonstrated that VPA0243 was the major virulence gene influencing survival and stays in the host for a long time [25]. VirK has a signal peptide for secretion through the Sec pathway into the periplasmic space [26]. VirK is recognized as a periplasmic protein that is necessary for the efficient secretion of plasmid-encoded toxins in Escherichia coli [27].

In many pathogenic bacteria, TolQ, one of the most widely distributed proteins, is one of the envelope proteins that constitutes the Tol system, contributing to colicin import and enhancing bacterial virulence. Abdelhamed et al. reported that the $\Delta t o l Q$ mutant in Edwardsiella ictaluri, a Gram-negative pathogen, had reduced virulence in catfish compared to wild type, which suggested that the Tol system was beneficial for Edwardsiella ictaluri virulence [28]. In addition, Tol family genes are also important in the pathogenic Gram-negative bacteria such as Haemophilus ducreyi, Vibrio cholerae [29], Salmonella enterica [30], and E. coli [31]. Previous reports showed that TolQ, TolR, TolA and TolB were essential to Salmonellae for keeping the outer-membrane closed and contributing to its virulence [32,33]. Except for the GPL pathway, STm Tol-Pal proteins may influence other biochemical and metabolic mechanisms to promote the virulence of many pathogenic bacteria.

VPA0202 encodes GGDEF domain, which plays a major role in synthesizing and degrading the nucleotide signal cyclic di-GMP (c-di-GMP) in some bacteria. A few years ago, it had been reported that c-di-GMP changed the pathogenicity of pathogenic bacteria and contributed to virulence. In V. parahaemolyticus, c-di-GMP-mediated regulation is balanced by regulating the ability of cell adhesion and movement, while changing the expression levels between the lateral flagellar and capsular polysaccharide genes [34]. A recent report on the mutant of Pseudomonas aeruginosa genes that encode GGDEF and / or an EAL domain proteins suggested that intracellular c-di-GMP levels made a difference for the factors related to virulence, for example, through the type III secretion system to regulate cytotoxicity [35].

VPA0318 encodes the outer membrane protein OmpV. Outer membrane proteins (OMPs) are widespread in Gram-negative bacteria [36] and are related to pathogenicity [37]. A previous study reported that outer membrane proteins could medicate the infection process including chemotaxis, motility through the mucous layer lining the intestinal wall, and survival of the gastric acid barrier [38]. Thus, outer membrane proteins are considered to be virulence factors. 


\section{Materials and Methods}

\subsection{Bacterial Strain and Growth Conditions}

The experimental strain V. parahaemolyticus CGMCC 1.1614 was obtained from China General Microbiological Culture Collection Center. Frozen stocks of V. parahaemolyticus were streaked onto 3\% sodium chloride tryptic soy agar (TSA-3\% NaCl) and incubated at $37^{\circ} \mathrm{C}$ overnight. A single colony was then transferred to $10 \mathrm{~mL}$ of $3 \%$ sodium chloride tryptic soy broth (TSB-3\% $\mathrm{NaCl}$ ) and cultured at $37^{\circ} \mathrm{C}$ for $6 \mathrm{~h}$ to the logarithmic growth phase.

\subsection{Antimicrobial Tests}

First, to select the strongest antibacterial effect of sulfur, we chose four isothiocyanate compounds to initially understand their antibacterial ability: benzyl isothiocyanate (BITC), phenyl isothiocyanate (PITC), 3-(Methylthio) propyl isothiocyanate (MTPITC) and isoamyl isothiocyanate (IAITC). The antimicrobial activity of sulfur compounds was measured using the diffusion assay method. Twenty milliliters of sterilized TSA-3\% NaCl was poured into a plate. After drying, the bacterical suspension was inoculated on the agar and mixed well. Afterwards, four filter papers were placed on the agar and $6 \mu \mathrm{l}$ sulfur compounds were added to the middle of paper, leaving one paper as a control. Then, the plats were cultivated at $37^{\circ} \mathrm{C}$ for $12 \mathrm{~h}$. The diameter of the inhibition zone (mm) of different sulfur compounds was measured with a Vernier caliper.

\subsection{RNA Extraction}

V. parahaemolyticus was treated with BITC at concentration of $1 / 4$ MIC (Q_BITC sample) or without BITC (C_BITC sample) as a control. Two samples of BITC were added in the logarithmic growth phase and shaken at $37^{\circ} \mathrm{C}$ and $150 \mathrm{rpm}$ for $6 \mathrm{~h}$. Total RNA from the two samples (C_BITC, Q_BITC sample) was extracted separately using the RNAprep Pure Cell/Bacterial Kit (Tiangen Biotech, Beijing, China) following its manual. Total RNA was quantified by measuring OD260 nm/OD280 $\mathrm{nm}$ ratio. RNA contamination and degradation was confirmed on $1 \%$ agarose gels $(w / v)$ stained with Goldview and visualized with a UV transilluminator (Versa Doc, Shanghai, China). All RNAs were stored at $-80{ }^{\circ} \mathrm{C}$ immediately until use.

\subsection{Library Preparation for Strand-Specific Transcriptome Sequencing}

Three micrograms RNA was used for the RNA sample preparations. Sequencing libraries were produced using NEBNext ${ }^{\circledR}$ Ultra $^{\mathrm{TM}}$ Directional RNA Library Prep Kit for Illumina ${ }^{\circledR}$ (NEB, Ipswich, MA, USA), and index codes were added to sequences. NEBNext First Strand Synthesis Reaction Buffer $(5 \times)$ was used with divalent cations at a high temperature to obtain fragmentation. The first-strand cDNA was synthesized by M-MuLV Reverse Transcriptase (RNase H, NEB, Ipswich, MA, USA) and random hexamer primer (NEB, Ipswich, MA, USA). The second-trand cDNA was synthesized with RNase $\mathrm{H}$ and DNA Polymerase I. The remaining overhangs were converted into blunt ends via exonuclease/polymerase activities. The AMPure XP system (Beckman Coulter, Beverly, MA, USA) was used to screen cDNA fragments of 150 200 bp in length to purify the library fragments. Then $3 \mu \mathrm{L}$ USER Enzyme (NEB, USA) was used with cDNA at $37{ }^{\circ} \mathrm{C}$ for $15 \mathrm{~min}$ followed by $5 \mathrm{~min}$ at $95{ }^{\circ} \mathrm{C}$ before PCR. Then, the reaction was carried out with Phusion High-Fidelity DNA polymerase kit (NEB, Ipswich, MA, USA), Universal PCR primers and Index (X) Primer. Finally, the Agilent Bioanalyzer 2100 system (G2939B, Agilent Technologies, Palo Alto, CA, USA) was used to assess products and library quality.

\subsection{Bioinformatic Analysis}

Clean data (clean reads) were acquired by removing reads including adapter, reads containing low quality reads and ploy- $N$ from raw data. In the meantime, GC content, Q20 (percentage of 
bases with a Phred value $>20$ ) and Q30 (percentage of bases with a Phred value $>30$ ) were obtained. All downstream data were analyzed based on the clean data with high quality. FPKM, expected number of Fragments Per Kilobase of transcript sequence per Millions base pairs sequenced, as accounts for the influence of gene length for the read count and sequencing depth. Thus, it is the method for evaluating gene expression. Gene Ontology (GO) enrichment analysis of differentially expressed genes was performed using the GOseq R package, which can correct the gene length bias. GO terms with adjusted $p$-values below 0.05 were thought to be significantly enriched by differentially expressed genes. KEGG is a database resource for comprehending high-level functions and tools of the biological system from molecular-level information. We employed KOBAS software to verify the statistical enrichment of differentially expression genes in KEGG pathways.

\section{6. qRT-PCR Validation of Differentially Expressed Genes}

In order to verify differential gene expression, total RNA was treated with the PrimeScript ${ }^{\mathrm{TM}}$ RT Reagent kit with gDNA Eraser (TaKaRa, Dalian, China) to remove genomic DNA and reverse transcribed. SYBR ${ }^{\circledR}$ Premix Ex Taq ${ }^{\mathrm{TM} I I}$ (TliRNaseH Plus) (Takara, Dalian, China) was used for qRT-PCR following the manufacturer's instructions. The $16 S$ rRNA gene was used as an endogenous gene and the differential gene expression level was assessed using the $2^{-\Delta \Delta C t}$ method [39]. All reactions were carried out by using annealing temperature of $58^{\circ} \mathrm{C}$ and a melting curve was obtained between 60 and $95^{\circ} \mathrm{C}$. All gene-specific primers were designed with Primer 5.0 software (PREMIER Biosoft, Palo Alto, CA, USA) and listed in Table 1.

\subsection{Statistical Analysis}

Data are shown as mean \pm standard deviation $(n=3)$. Student's t-test was used to assess the difference between two groups. Differences between each sample were recognized to be statistically significant at the $p<0.05$ level.

\section{Conclusions}

The use of natural products can provide a new way to prevent and treat microorganisms in the food industry. This study is the first to report on the antimicrobial effect of BITC on $V$. parahaemolyticus at the gene level via transcriptome analysis, indicating a regulation of different virulence genes that leads to destabilization of bacteria. Further studies will focus on the screening of differentially expressed genes in a large scale. The protein level expression of the target genes as well as gene knock out studies should be performed to validate intricate regulations in biochemical and physiological properties of key targets.

Author Contributions: Methodology, J.S.; formal analysis, H.-Y.W. and K.-X.L.; data curation, Y.W. and Q.-Q.Z.; writing-original draft preparation, J.S. and G.-L.Z.; writing-review and editing, G.-L.Z. and H.-M.H.; supervision, H.-M.H.; funding acquisition, G.-L.Z. All authors discussed the results and approved the final manuscript.

Funding: This research was funded by the National Natural Science Foundation of China, grant number 31571888 .

Conflicts of Interest: The authors declare no conflict of interest.

\section{References}

1. Shen, X.; Su, Y.C. Application of grape seed extract in depuration for decontaminating Vibrio parahaemolyticus in Pacific oysters (Crassostrea gigas). Food Control 2016, 73, 601-605. [CrossRef]

2. Lovell, C.R. Ecological fitness and virulence features of Vibrio parahaemolyticus in estuarine environments. Appl. Microbiol. Biotech. 2017, 101, 1781-1794. [CrossRef] [PubMed]

3. Song, X.; Ma, Y.; Fu, J.; Zhao, A.; Guo, Z.; Malakar, P.K.; Pan, Y.; Zhao, Y. Effect of temperature on pathogenic and non-pathogenic Vibrio parahaemolyticus biofilm formation. Food Contr. 2016, 73, 485-491. [CrossRef] 
4. Su, Y.C.; Liu, C. Vibrio parahaemolyticus: A concern of seafood safety. Food Microbiol. 2007, 24, 549-558. [CrossRef] [PubMed]

5. Zhu, M.Y.; Li, W.; Dong, X.; Li, M.S. Benzyl-isothiocyanate induces apoptosis and inhibits migration and invasion of hepatocellular carcinoma cells in vitro. J. Cancer 2017, 8, 240-248. [CrossRef] [PubMed]

6. Liu, Q.; Meng, X.; Li, Y.; Zhao, C.N.; Tang, G.Y.; Li, H.B. Antibacterial and antifungal activities of spices. Int. J. Mol. Sci. 2017, 18, 1283-1321. [CrossRef] [PubMed]

7. Feng, L.; Zhang, K.; Gao, M.; Shi, C.; Ge, C.; Qu, D.; Zhu, J.; Shi, Y.; Han, J. Inactivation of Vibrio parahaemolyticus by aqueous ozone. J. Microbiol. Biotech. 2018, 28, 1233-1246.

8. Morse, M.A.; Zu, H.; Galati, A.J.; Schmidt, C.J.; Stoner, G.D. Dose-related inhibition by dietary phenethyl isothiocyanate of esophageal tumorigenesis and DNA methylation induced by $N$-nitrosomethylbenzylamine in rats. Cancer Lett. 1993, 72, 103-110. [CrossRef]

9. Smolinska, U.; Morra, M.J.; Knudsen, G.R.; James, R.L. Isothiocyanates produced by Brassicaceae species as inhibitors of Fusarium oxysporum. Plant Disease 2003, 87, 407-412. [CrossRef]

10. Dufour, V.; Stahl, M.; Baysse, C. The antibacterial properties of isothiocyanates. Microbiology 2015, 161, 229-243. [CrossRef]

11. Nakano, M.; Takahashi, A.; Su, Z.; Harada, N.; Mawatari, K.; Nakaya, Y. Hfq regulates the expression of the thermostable direct hemolysin gene in Vibrio parahaemolyticus. BMC Microbiol. 2008, 8, 155. [CrossRef] [PubMed]

12. Shirai, H.; Ito, H.; Hirayama, T.; Nakamoto, Y.; Nakabayashi, N.; Kumagai, K.; Nishibuchi, M. Molecular epidemiologic evidence for association of thermostable direct hemolysin (TDH) and TDH-related hemolysin of Vibrio parahaemolyticus with gastroenteritis. Infect. Immun. 1990, 58, 3568-3573. [PubMed]

13. Banu, S.F.; Rubini, D.; Murugan, R.; Vadivel, V.; Gowrishankar, S.; Nithyanand, P. Exploring the antivirulent and sea food preservation efficacy of essential oil combined with DNase on Vibrio parahaemolyticus. LWT 2018, 95, 107-115. [CrossRef]

14. Tan, X.J.; Qin, N.; Wu, C.Y. Transcriptome analysis of the biofilm formed by methicillinsusceptible Staphylococcus aureus. Sci. Rep. 2015, 5, 11997. [CrossRef] [PubMed]

15. Pieta, L.; Escudero, F.L.G.; Jacobus, A.P. Comparative transcriptomic analysis of Listeria monocytogenes reveals upregulation of stress genes and downregulation of virulence genes in response to essential oil extracted from Baccharis psiadioides. Ann. Microbiol. 2017, 67, 479-490. [CrossRef]

16. Aagesen, A.M.; Phuvasate, S.; Su, Y.C. Characterizing the adherence profiles of virulent Vibrio parahaemolyticus isolates. Microb. Ecol. 2018, 75, 152-162. [CrossRef] [PubMed]

17. Wang, R.Z.; Zhong, Y.F.; Gu, X.S. The pathogenesis, detection, and prevention of Vibrio parahaemolyticus. Front. Microbiol. 2015, 6, 144. [CrossRef] [PubMed]

18. Sofrata, A.; Santangelo, E.M.; Azeem, M.; Gustafsson, A. Benzyl isothiocyanate, a major component from the roots of Salvadora persica is highly active against gram-negative bacteria. PLoS ONE 2011, 6, e23045. [CrossRef] [PubMed]

19. Hong, L. Analysis of the collective food poisoning events in Shanghai from 1990 to 2000. Chinese J. Nat. Med. 2003, 5, 17-20.

20. Romocastillo, M.; Andrade, A.; Espinosa, N. EscO, a functional and structural analog of the flagellar FliJ protein, is a positive regulator of EscN ATPase activity of the enteropathogenic Escherichia coli injectisome. J. Bacteriol. 2014, 196, 2227-2241. [CrossRef] [PubMed]

21. Claret, L.; Calder, S.R.; Higgins, M.; Hughes, C. Oligomerization and activation of the FliI ATPase central to bacterial flagellum assembly. Mol. Microbiol. 2010, 48, 1349-1355. [CrossRef]

22. Cornelis, G.R. The type III secretion injectisome. Nat. Rev. Microbiol. 2006, 4, 811-825. [CrossRef] [PubMed]

23. Haiko, J.; Westerlund-Wikström, B. The role of the bacterial flagellum in adhesion and virulence. Biology 2013, 2, 1242-1267. [CrossRef] [PubMed]

24. Detweiler, C.S.; Monack, D.M.; Brodsky, I.E.; Mathew, H.; Falkow, S. virK, somA and rcsC are important for systemic Salmonella enterica serovar Typhimurium infection and cationic peptide resistance. Mol. Microbiol. 2010, 48, 385-400. [CrossRef]

25. Spencer, H.; Karavolos, M.H.; Bulmer, D.M.; Aldridge, P.; Chhabra, S.R.; Winzer, K.; Williams, P.; Khan, C.M.A. Genome-wide transposon mutagenesis identifies a role for host neuroendocrine stress hormones in regulating the expression of virulence genes in Salmonella. J. Bacteriol. 2010, 192, 714-724. [CrossRef] [PubMed] 
26. Ferreira, R.M.; Moreira, L.M.; Ferro, J.A.; Soares, M.R.; Laia, M.L.; Varani, A.M.; de Oliveira, J.C.; Ferro, M.I. Unravelling potential virulence factor candidates in Xanthomonas citri. subsp. citri by secretome analysis. Peer J. 2016, 4, e1734. [CrossRef]

27. Gabriela, T.P.; Lucia, C.D.A.; Humberto, L.M.; Ken, T.; Fernando, N.G. VirK is a periplasmic protein required for efficient secretion of plasmid-encoded toxin from enteroaggregative Escherichia coli. Infect. Immun. 2012, $80,2276-2285$.

28. Abdelhamed, H.; Lu, J.; Lawrence, M.L.; Karsi, A. Involvement of tolQ and tolR genes in Edwardsiella ictaluri virulence. Microb. Pathogenesis 2016, 100, 90-94. [CrossRef]

29. Heilpern, A.J.; Waldor, M.K. CTXphi infection of Vibrio cholerae requires the tolQRA gene products. J. Bacteriol. 2000, 182, 1739-1747. [CrossRef]

30. Bowe, F.; Lipps, C.J.; Tsolis, R.M.; Groisman, E.; Heffron, F.; Kusters, J.G. At least four percent of the Salmonella typhimurium genome is required for fatal infection of mice. Infect. Immun. 1998, 66, 3372-3377.

31. Judith, H.; Jr, R.J.; Meganm, T.; Jennifere, A.; Hshaw, W. Bacterial peptidoglycan-associated lipoprotein is released into the bloodstream in gram-negative sepsis and causes inflammation and death in mice. J. Biol. Chem. 2002, 277, 14274-14280.

32. Tamayo, R.; Ryan, S.S.; Mccoy, A.J. Identification and genetic characterization of PmrA-regulated genes and genes involved in polymyxin B resistance in Salmonella enterica serovar typhimurium. Infect. Immun. 2002, 70, 6770-6778. [CrossRef] [PubMed]

33. Paterson, G.K.; Northen, H.; Cone, D.B.; Willers, C.; Peters, S.E.; Maskell, D.J. Deletion of tolA in Salmonella Typhimurium generates an attenuated strain with vaccine potential. Microbiology 2009, 155, 220-228. [CrossRef] [PubMed]

34. Kim, Y.K.; Mccarter, L.L. ScrG, a GGDEF-EAL protein, participates in regulating swarming and sticking in Vibrio parahaemolyticus. J. Bacteriol. 2007, 189, 4094-4107. [CrossRef] [PubMed]

35. Lim, B.; Beyhan, S.; Yildiz, F.H. Regulation of Vibrio polysaccharide synthesis and virulence factor production by CdgC, a GGDEF-EAL domain protein, in Vibrio cholerae. J. Bacteriol. 2007, 189, 717-729. [CrossRef] [PubMed]

36. Xiong, X.P.; Wang, C.; Ye, M.Z. Differentially expressed outer membrane proteins of Vibrio alginolyticusin response to six types of antibiotics. Mar. Biotech. 2010, 12, 686-695. [CrossRef]

37. KIM, M.S.; JIN, J.W.; JUNG, S.H. Genetic variations of outer membrane protein genes of Vibrio harveyi isolated in Korea and immunogenicity of OmpW in olive flounder, Paralichthys olivaceus. J. Fish. Mar. Sci. Educ. 2015, 27, 1508-1521.

38. Taylor, R.K.; Manoil, C.; Mekalanos, J.J. Broad-host-range vectors for delivery of TnphoA: Use in genetic analysis of secreted virulence determinants of Vibrio cholerae. J. Bacteriol. 1989, 171, 1870-1878. [CrossRef]

39. Livak, K.J.; Schmittgen, T.D. Analysis of relative gene expression data using real-time quantitative PCR and the 2(-Delta Delta C(T)) method. Methods 2001, 25, 402-408. [CrossRef]

Sample Availability: Samples of the compounds are not available from the authors.

(C) 2019 by the authors. Licensee MDPI, Basel, Switzerland. This article is an open access article distributed under the terms and conditions of the Creative Commons Attribution (CC BY) license (http:/ / creativecommons.org/licenses/by/4.0/). 